\title{
El trabajo femenino en Mallorca. La labor de la mujer en la actividad marítima de la primera mitad del siglo XIV
}

\author{
Antonio ORTEGa Villoslada
}

\section{RESUMEN ABSTRAC}

La participación de la mujer en la actividad maritima y comercial de Mallorca está todavia pendiente de estudio. Este trabajo muestra algunos trazos de dicha actividad tanto en el puerto de Palma como en los puertos atlánticos.

PALABRAS CLAVE Mujer, Edad Media, Comercio, Mallorca
The participation of the woman in the marine and commercial activity of Majorca is still pending of study. This work as much shows some outlines of this activity in the port of Palma like in the Atlantic ports

\section{KEY WORDS}

Woman, Average Age, Commerce, Majorca

\section{INTRODUCCIÓN}

Los estudios sobre la mujer en la sociedad medieval no llegan a España hasta la década de los 80 y desde entonces han experimentado un notable avance ${ }^{1}$. No voy a entrar en la evolución que han tenido ni en las interminables discusiones metodológicas que continúan generando ${ }^{2}$ pues lo que presentan estas páginas es un breve retazo sobre la actividad de las mujeres mallorquinas iigadas, de una $u$

1 Don José-Luis Martin se interesó por la mujer medieval en trabajos como Amor y Sexo en la España Medieval o Mujer y refranero. Este trabajo forma parte de la tesis doctoral que me dirigía bajo el título: El Reino de Maliorca y el mundo atlántico.

2 Una exposición sobre este asunto se puede ver en MORANT, Isabel. Historia de la mujeres e historia: innovaciones y confrontaciones. 
otra forma, al principal medio económico insular, como era el marítimo, ya que tradicionalmente no se ha prestado la debida atención a su papel en el comercio ${ }^{3} \mathrm{y}$, en especial, en el comercio atlántico, ámbito no estudiado hasta la fecha.

Para que la participación femenina fuese efectiva era necesario el acceso a una formación que, a inicios del siglo xIv, secundaban muy pocos. Además, aprender a leer era visto por muchos como algo incluso pernicioso. Si en Cataluña Cerveri de Gerona o Jaume Roig censuraban en sus poemas a la mujer ilustrada, en Italia autores como Felipe de Novara o, posteriormente, Paolo da Certaldo consideraban que la lectura sólo podia conducirla al peor de los males. Afortunadamente esta actitud contaba con opositores que destacaban la importancia de su instrucción ${ }^{4}$. De todos ellos me interesa resaltar a Francisco de Barberino quien recomendaba, a inicios del siglo XIV, en su tratado Del reggimento e costumi di donna que las mujeres leyesen por un motivo eminentemente práctico como era el de tener capacidad para administrar los bienes de sus maridos. Parece ser ésta la postura adoptada en Mallorca, ya desde antiguo, respecto a la educación femenina pues las propias franquezas y privilegios de las islas contemplaban su fundamental papel en el gobierno de las propiedades y negocios del marido ausente por actividad comercial o maritima ${ }^{5}$.

\section{LAS MUJERES EN EL COMERCIO MARITIMO ATLÁNTICO}

Es así que esta participación estaba regulada por ley: ...Ponit quod de obseruancia et consuetudine terre Maiorice et curiarum est quod nauigantes et negociantes absentes se per negociis suis ab hac terra Maiorice frequentius constituunt procuratores uxores suas seu alias mulieres honestas et (conuenientas) generaliter ad negocia sua ${ }^{6}$.

Estas mismas costumbres permitían que ellas a su vez pudieran nombrar procuradores para ponerlos al frente de los negocios: ...Dantes et concedentes predictis mulieribus quod posint alios procuratores substituere ad negocia et etiam constituere ad licens cum plena postestate; et de hiis est veritas et publica fama.

3 Peroud, Regine. La mujer en el tiempo de las catedrales, p. 210. Madrid, 1982

- En La dona en Francesc Eiximenis, p. 35 a 43, los autores resumen las respectivas posiciones de los diversos escritores ante la educación femenina

${ }^{5}$ Carmen BATLle, en Noticias sobre la mujer catalana..., estudió la participación de las mujeres en la vida laboral barcelonesa del siglo xili en la que destacaba su colaboración en los negocios de sus maridos y la continuidad de éstos una vez fallecido éste, asi como la aptitud femenina en este campo y el talento en su administración.

${ }_{6}$ A.R.M A.H-438, fol. $80 \mathrm{v}$. El 16 de diciembre de 1338 se produce un pleito, por unas deudas, entre Mariana, mujer del mercader Guillermo Cima, y Pedro Pons en el que se recuerda estas costumbres. En este pleito el mercader Agustín Cima nombrará procuradora a su abuela: ...ltem, ponit quod Gosti cima, ciuis Maioricarum, quando recessit ab hac terra constituit generaliter procuratorem suam ad negocia Domina Matianam, uxorem Guillelmi cima, quondam auunculi dicti Gostini. Dando sibi quod posset alium uel alios procuratores substituere ad negocia et ad literis constituere cum plena potestatem... 
Sin embargo, la relación de las mujeres mallorquinas con el comercio marítimo es un campo todavía pendiente de estudio. Si en el ámbito mediterráneo se han realizado algunas aproximaciones ${ }^{7}$, no ocurre lo mismo hacia el mundo atlántico. Veamos algunos rasgos de su actuación en dicho comercio.

En el rol femenino se incluia la preparación de los vástagos para la actividad comercial y, en ocasiones, la desagradable tarea de tener que reivindicar los bienes de alguno de ellos cuando fallecían lejos de Mallorca durante el desarrollo de su oficio. Este es el caso de Elisenda que tendrá que reclamar los bienes de su hijo, que había muerto en Lisboa durante una travesía a La Rochela ${ }^{8}$. Efectivamente, en mayo de 1295 Nicolás Tarida y Daniel Delatorre, mercaderes mallorquines, inician su viaje ${ }^{9}$. Parten de Mallorca, en la coca de Daniel Can, con pimienta, azafrán y doblas rumbo a Sevilla donde venderán estas mercancias. Allí, junto al italiano Luquin Dugo, compran una coca, marchan a Alaraix para estibarla de grano y ponen proa a La Rochela. En el trayecto recalan en Lisboa donde, después de legar sus bienes a su compañero, fallecerá Nicolás Tarida. Luquin Dugo y Daniel Delatorre prosiguen su ruta y hacen escala en Santander, lugar en el que se les unirá el italiano, y ciudadano de Mallorca, Lanfranco Negro. Pero la coca no continuará viaje pues tanto ésta como el grano serán vendidos en el mismo Santander.

Once años más tarde, encontramos a la madre de Nicolás Tarida reclamando a Lanfranco Negro la parte correspondiente de la venta. Para ello, pide al lugarteniente de Mallorca que se realice en Sevilla una investigación sobre este asunto a través de Cammo de Génova, cónsul genovés en Sevilla, e interroguen a Daniel Delatorre y a Luquin Dugo: ...Sapia la uostra discrecio per les presens que denant nos son estats e comparegutz en bernat net e Narnau de cases e en Francesc de comeles, arbitres comunament eletz (...) questio ques mena entre ladona Nalicsenda tarida, mare den Nicholau tarida duna part sa enrera, e lafrancho negre, Jenoues ciutada de Malorches daltra, e requeriren nos que per nostre offici vos deguessem trametre alcuns duptes en queson duptans quant adetermenar clarament (quant) alodit contrast; los quals duptes volen e demanen que sien esclaritz e determenatz clarament aixi con son enueritat dasso que hi sapia per luquni dugo, Jenoues companyo del dit Nicholau tarida sa enrera, e den Daniel dela torra odela uns daquels ${ }^{10} \ldots$

7 Un reciente estudio sobre el papel de las mujeres genovesas residentes en Mallorca ha sido publicado por TUDELA VILLALONGA, Luis. Les dones dels mercaders genovesos i mallorquins a la primera mejtat del segle XIV.

A.R.M. L.R-2, fols. $141 \mathrm{v}-143 \mathrm{v}$. Entre las múltiples cuestiones a tratar, debia interesarse por el flete del grano entre Alaraix y Santander: ...fou pagat nolit alseu forment ne quant nolit fo ne com se parti e quant noch lidit $L$ (uquin) (...) dela sua part del nolit e si hac negun altre nolit de neguna altra persona de forment (e) (de) (...) partent delaraix tan fins a Sent ander. to si reebe lodit forment Laffrancho...-143r-

`En un trabajo anterior documentaba la presencia de mercaderes de la Corona de Aragón en la ruta de cabotaje hacia Flandes ai menos desde los años 30 del siglo xIv. Gracias a este pleito constatamos la temprana participación de mercaderes y navegantes mallorquines en esta ruta ya desde finales del siglo XIII. ORTEGa VIlloslada, A. Viajes a Flandes e Inglaterra. ¿Cabotaje o "recta via"? Espacio, Tiempo y Forma, Serie, III, Historia Medieval, tomo 16, págs. 229-249.

10 A.R.M. L.R-2, fol. $141 \mathrm{v}$ 
Otra de las facetas bien conocidas de la actividad femenina radica en los requerimientos efectuados por las esposas y madres de los mercaderes cuando caian presos. Tal es el caso de Bienvenida, quien ruega al lugarteniente Roger de Rovenach que interceda en la liberación de su marido Antonio Maestre, patrón y propietario de nave, y de otros mercaderes mallorquines, apresados en 1341 en aguas de Portugal por una galeota de Arcila y encarcelados en Marruecos: ... a instancia e supplicatio den Anthoni salurada e den ffrances(sic) botar e den Arnau de quadres, en Pere de beyluei e den bonanat ferrer, mercaders e ciutadans de malorches, e dena benuenguda, muller den Anthoni maestre, e per part den Berenguer borreyl. Erequeri la uostra altea que deguessetz fer restituir e deliurar lo vaxel den Anthoni masestre que fo pres en los mars del Rey de portugal per una galiota de Arcilla que menaua Haharon en lo qual vaxel hauia homens de malorcha e mercaderies dels dits soplegants; los homens que heren en lo dit vaxel sotzmeses de nostre senyor lo Rey son aquestz, soes, Anthoni maestre, Berenguer borreyl; eles mercaderies que en lodit vaxel eren son aquestes... ${ }^{11}$.

Ante el estado de desamparo en que quedaban sumidas las familias de los mercaderes cautivos, los monarcas mallorquines instituirán ayudas para su redención, vendiendo incluso algunos esclavos para sacar fondos: ... Item, deliuraren de manament del Senyor Rey a alcunes dones de Malorques, per trer lurs marits de catiu que eren preses aSalcudia, III Sarrahins que foren aprehats enlo compte dela armada del noble en Gisbert de castel nou........ C libras ${ }^{12}$.

También en el apartado financiero observamos a las mujeres actuando con plena libertad. En algunos casos, como demandantes cuando se producen comandas fallidas, como en el caso de Jacomina, esposa de Guioto Asberguerii, que denuncia a Osbert Dast por la pérdida de una comanda a tratar en Sevilla. Debía vender unas mercancías, valoradas en 8 libras de Mallorca, y con los beneficios comprar paños de Arrás y doblas de oro. Sin embargo esta operación no se podrá realizar pues Osbert Dast será, según dice él, apresado ${ }^{13}$.

Por otro lado, participan activamente en el comercio atlántico a través de las comandas. Veamos algunos ejemplos. En 1349 es Blanca, hermana y esposa de mercaderes, quien da al mercader Francisco Argelí una comanda de 150 libras para negociarlas en Nife en su próximo viaje con la coca den Sala ${ }^{14}$.

1 A.R.M. A.H-2, fols. 149r-150r. Aprovecha esta misma carta a Abu-l-Hasán, rey de Marruecos, para solicitar la restitución de varios leños mallorquines apresados por hombres de Ceuta en aguas cercanas a Mallorca - uno de ellos en la isla de La Dragonera- y una coca, en viaje a Tedelis, asaltada por hombres de Argel. También reclama la liberación de un mercader mallorquín capturado en un leño de castellanos que navegaba de Sevilla a Tarifa.

12 A.R.M. R.P.3038, fol. 54v.

13 A.R.M. Suplicaciones, n. ${ }^{\circ}$, fols. $125 r-127 v$. El pleito se desarrolla durante el mes de julio de 1300 y en él se hace referencia al encarcelamiento, tres años antes, de mercaderes catalanes y genoveses en el puerto de Salé: $-125 v-.$. Item, ponit dictus obertus dast quod ipse III anni sunt elapsi, parum plus uel minus, quod hic obertus et omnes xristiani qui tunc venerant de Xibilia apud Sale, tam catalani quam januenses, fuerunt capti per dominationem dicti loci de Sale..

14 A.C.M. Protocolos notariales, n. 14566 , fol. $95 \mathrm{~V}$ 
En 1335 Gerarda entrega a Miguel Vilagne, de Mallorca, una comanda de 7 libras y 3 sueldos invertidas en 20 espuertas de higos para venderlas en Sevilla por medio de alguna coca castellana ${ }^{15}$.

Unos años antes, en 1327, doña Sancha envía a Arzila, en dos cocas diferentes, una remesa de telas, por un total aproximado de 187 libras de Mallorca, para que las gestione el mercader mallorquín $\mathrm{G}$. Mel, residente en dicha localidad marroquí. La muerte de este factor provocará el pleito entre Pedro Safont y Pedro Casas - socios de G. Mel-y doña Sancha, que reclamará la devolución o restitución de estos bienes: ...Com los dits marmessors contredige de donar e de restituir ala dita dona na Sanxa les dites coses, o la extimacio dequeles, perso demane e requeri la dita Sanxa los dits marmassors per uos altres senyors arbitres esser condempnats en dar e en pagar aladita dona na Sanxa les dites altres cozes oles extimacions daqueles... ...pose e enten a prouar que sil dit G. mel sa enrere reebe ascuns draps o xamelots o badenchs dela dita dona aqueles tremes, eel preu reebe e aquel preu posa a converti enla companyia que ere entrel dit G. mel, en $P$. safont e en $p$. de cases, e enlos fets e negocis de la dita companyia..... Item, pose $e$ enten a prouar que sil dit $G$. mel reebe alcuns draps o xamelots o badenchs de la cita dona, aquells o partida daquels romangeren en Arzila en poder dels faedors dela dita companyia, e moltes persones veeren aquels... ${ }^{16}$

Pero no sólo comerciaban desde la isla sino que también compartian con sus parejas la residencia en lugares lejanos como le ocurrió a Catalina -en este momento esposa de Guillermo Sescases- quien en 1330 residía en Arzila con G. Mel que, como hemos visto, fallecerá en el puerto marroquí. Este mercader tenía como ayudante a Perico Sacanal con el que mantendrá un pleito a causa del dinero que le debe por su trabajo de 3 años ${ }^{17}$. Durante el pleito se pedirá declaración

15 A.C.M. Protocolos notariales, n. 14545, fol. 59r: ... Sit omnibus notum quod ego Michel vilagne, ciuis Maioricarum, confiteor in comanda tenere auobis Geralda, uxori quondam berenguer morga, conciuum meum et vestris, septem libras et tres solidos monete regalium Maioricarum minutorum quas auobis habui et recepi emerciatas in viginte sportis ficarum... .. dictam comandam promito mecum portare in quadam cocha castellana apud xibilia et ab inde... ...causa mercaterie redeundo Maioricarum, in quo viagio promito habere comanda vendere et precium inde habitum emerciare...

16 A.C.M. Protocolos notariales, n. 14559 , fols. $83 r-85 r$. Los socios del mercader fallecido se niegan a pagar la deuda contraida con doña Sancha y para ello alegan antiguos privilegios y costumbres entre los mercaderes mallorquines: ... Item, pose e enten a prouar que tot e cascuna es e es estada lonch de temps en maiorques oberuada entre mercaders que si alcun mercader companyo o faedor dalcuna companyia procurant la dita companyia reeb alcunes mercaderies daltres mercaders qui no sien dela companyia, les quals al preu daqueles comanem o son innuertides enla dita companyia e els bens daquela son tenguts en satisfer les dites mercaderies als dits mercaders qui les han trameses, los hereus del dit companyo mort no son tenguts deles altres bens seus en asso en alcuna cosa, non poden esser demanatz ne couenguts maiorment com los hereus del dit companyo no demanen ne volen axi si no so quel dit companyo auia enla dita companyia...

17 A.C.M. Protocolos notariales, n. 14557 , fols 20r-23v. Perico Sacanal reclama a Juan Pont, ejecutor testamentario de G. Mel, el salario correspondiente a los tres años que estuvo trabajando para él como ayudante en Arcila. Pide el pago de 6 doblas de oro anuales -algunos llegarán a cobrar hasta 10 doblas de oro anuales, según su valia- pero por sentencia únicamente cobrará 4 doblas anuales al entender el juez que no realizaba trabajos propios de ayudantes expertos y se limitaba a los asuntos domésticos: dien quel dit perico parti de mayorques axi com a macip e seruicial del dit $G$. e ana ab ell ales parts de 
a Catalina quien confirma que estuvo viviendo con este mercader en Arzila y que cuando partió, Perico Sacanal todavía se quedó un año más al servicio de G. Mel: ...Caterina, muler den $G$ ses cases... ... dix que sap azo solament que be ha $V o V I$ anys passats que aquesta estaue ab lodit $G$. mel a arzila enlo fondech del dit $G$., $e$ estant aqui vee lodit perico e tant ab lo dit $G$ per seruicial faent les faenes del alberch axi com par cuynar, agranar e ajudant en altres coses segons son poder per II anys continuament, e oy dir al dit G. que el tenie lo dit perico a soldada mas li oy dir quant li donaue lany... ... dix encara quel dit perico estech ab lodit $G$ de puis que aquesta parti dartzila ben per I any. Item, dix que estant aquesta ensemps ab lo dit $G$ en 10 dit fondech, vee quel dit perico senfeloni ab lo dit $G$. en axi quel dit $G$ lo feri, el dit perico denana lauors aldit $G$. mel VII dobles que dehie que tenie sues les quals lo dit $G$ noli negaue ans li dehie: "no ages paor, que nom tendre res del teu que mes tendonare, mas volire que ten profitasses"

Además, las mujeres actuaban en otros asuntos relacionados con el puerto y en concreto con la administración portuaria mallorquina. Es el caso de Na Granada quien en 1318 es propietaria del establecimiento donde tienen su oficina los guardianes del puerto de Palma y por el cual le deben pagar cada año el alquiler del local: ...ltem, ala ciona na Granada, per cens que pren sobra ladita butiga e $I^{a}$ que es al carrer dels exauegers. I libra ${ }^{18}$.

\section{LAS MUJERES EN LAS ATARAZANAS}

Es precisamente en el ajetreo portuario donde encontramos otra de las facetas del papel femenino en la actividad marítima mallorquina, como es su vinculación laboral con las atarazanas.

\footnotetext{
barberia, soes assaber, al loc darzila hon serui a ell e feu sos seruici axi com a macip seu continuament per III anys e mes hon com lodit perico meresque auer per son seruici per los dits III anys XVIII dobles dor e molt mes... ... Primerament enten a prouar lodit en $P$. sa canal que parten lo dit $G$. de mallorques $e$ anant ales parts de barberia, soes assaber, en arzila, e son VI anys passats e mes, lo dit $G$. sen mena ab si per macip en perico sa canal, fil del dit $P$., prometenli de donar bona soldada segons quels altres mercaders han acustumat de donar a macips ques seruesquen enles parts de barberia...... e vee que fahie totes les faenes enlo fondech axi com macip, soes assaber, pastar, cuynar, fer litz, agranar e altres faenes de casa, mas no sap sol de $G$. mel promes de donar ael soldada o no... ... mas be es cert que fort sotil macip o fadri qui aye poder de fer faenes de casa pren VI dobles lany $e$ any quen prenen VIII $\mathrm{X}$ segons la espertea del macip.

18 A.R.M. R.P-3038, fol. 54v. Durante el periodo de la Casa de Mallorca los guardianes del puerto tendrán su oficina y almacén en esta tienda sita en una alhóndiga que habia sido propiedad de su padre Jaime Granada. El 26 de julio de 1280 na Granada, esposa de Roberto de Pulcro Vicino, alquila a Berenguer Vila el patio de una casa que está en este recinto. Lo mismo hace con Arnau Segarra. La descripción sitúa esta alhóndiga al lado del mar: ...et ex alia parte in litum maris... A.R.M. E.C.R-350, fol. 21v. El 13 de marzo de 1282, encontramos una nueva referencia a su situación cuando el botero $R$. Font alquila al remolar Simón Tona un taller que está fuera de las murallas y junto al muelle de la ciudad. Si en uno de los lados limita con la propia muralla, en otro linda con el obrador de Na Granada: ...R. de Fonte, boterius, per me et meos dono (et) stabilo tibi Simoni Tona, remolaris, et tuis propio unum operatorium cum solerio de super quod habeo extra Ciuitatem Maiorice juxta mollum eiusdem Ciuitatis... ...affrontat ex una parte in Domibus Asselmi de Dulcia, et ex alia parte in muro dicte Ciuitatis, et ex alia parte in operatorio de na Granada... A.R.M. E.C.R-350, fol. 133v.
} 
Algunas mujeres se han convertido en proveedoras de material náutico, tal y como le ocurre a Bienvenida que en 1324 continuará con el negocio de su marido abasteciendo a la atarazana: ...E nos devem aeyl $G$ (...) per wos ala dona nabeuenguda, muler que fo den Damau ses portes, e foren per estopa e clauo aladob de les Galeas del pariatge, dimecres a XII de juyn de MCCCXXIIII, en comte deladita dona. LXXII libras, XVII sous, $\| /^{19}$.

En 1326, Na Portes provee, junto a P. Calafat, P. Mandri y otros, lo necesario para la reparación de una galera de 104 remos y 1 leño armado de 84 remos: ...pagam an Corral Scorba ean p. calafat e an P. mandri e ana portas e adal(tres) persones per stopa e pega quen prezen aops del adob dels ditz lenys e per altres (mess)ions necessaries per los ditz lenys axi con se conten per partides e per menut en $I^{a}$ plagina el libre manual en VIII cartes.........XXII libras, XVI sous, $X$. Item, pagam an $P$. mascager, ferrer, $e$ an $P$. calafat e aladona na Portes per $I^{m} C$ agutz (...) ombre e per altre clauo aops dels ditz lenys axi con se conten el dit manual (per) partides e asumat en $I^{2}$ plagina en IX cartes...........IIII libras, XIIII sous, $X^{20}$ Y colaborará con $\mathrm{Na}$ Galeza, en el aporte de material para la construcción de dos nuevas galeras del pariatge: ...pagam an Bonanat lobet e ala dona na Galeza per compra quen fem dalquitra e de pega e daltres persones laqual presem per partides e adiuerses persones preses aops deles dites Galeas axi con se conten.... .........XXVIII libras, VII sous. Item, pagam aladona na Portes per peyls de lanades quen presem e per compra que fem de coto a embandar les cubertes e per altres messions fetes perles dites Galeas, axi con se conten. IIII libras, III sous, $\| \mathbb{R}^{1}$.

También intervienen en los trabajos de mantenimiento de los distintos elementos y materiales náuticos. Así, en 1321 Corral Escorba pagará algo más de 11 libras a las mujeres que han hecho estopa: Item, que ha pagades lo dit en corral scorba atotes les fembres que an feyta lestopa, segons que es conuengut en $I^{a}$ sedula donada perel de loger deles dites fembres largament, e amb la libra, VI sous, $V I$ diners que ach de mesions de morques........XI libras, $X$ sous, $V I^{22}$.

19 A.R.M. R.P. 3382 , fol. $13 \mathrm{v}$. En el $36 \mathrm{r}$ le vueiven a consignar nuevas cantidades por el material empleado en la preparación de la flota de Cerdeña: ... Deu la dona Nabeuenguda, muler qui fou den Dalmau ses portes, que li donam comtans, diyous a X iorns de Maig de lany de MCCCXXIIII, e fo per pegua e estopa e estoperals quen present que pres per eyla Petro casteyo, comtans.................XXX libras, III sous. 1. ...E nos devem aeyla per XXXXVII Milers destoperols quen compram aops del adop deles Galeas de Cerdenya, arao de XI sous, VI diners 10 Miler............XXVII libras, VI cent XCIII libras destopa prima e grossa quen compram aops del dit adop a rao de XXXVI sous lo cent, munta................XXXXVIII libras, VIIII sous

20 A.R.M. R.P-3051, fol 49 r.

21 A.R.M. R.P-3051, fol. $51 \mathrm{r}$.

22 A.R.M. R.P-3382, documento suelto. En el folio $38 \mathrm{v}$ está el asiento correspondiente añadiendo algo más de una libra en la confección de estopa....Item. li deuem per so que paga a fembres qui feeren la estopa en la darassana ab XXVI sous, VI diners que costaren morques a fer la dita estopa. segons que axi matex mostra per escrit en I paper que es assi...............XI libras, $X$ sous, VI. liem, lideu hom per messions que feu en fembres que feeren estopa que era romasa, segons que en l escrit quens dona es. contengut.................. I libra. VIII sous, VIIII. 
El repaso de las velas era otra de las funciones femeninas, tal y como aparece reflejado en un cuadernillo de 1328 donde se recogen los gastos realizados por el guardián de la atarazana ${ }^{23}$. En él se especifican las misiones correspondientes a las mujeres: Item, pagam que munten les mecions (...) Jornals de fembres per cozir les veles dela Galea natzarn e del leny Sent Saluador e per compra defil deueles e den palomar (... ......... VIII libras; asi como la confección y arreglo de sacos: e pagam per (iornals) de fembres per cozir LX Sachs nous, e per cozir e per adobar CX Sachs vels e per fil de ueles comprar als sachs vels Acozir, e per altres mecions fetes per(la) (...) (da)rassana, axi conseconte..... XV libras.

Item, Pagam per temps esdeuenidor per Jornals defembres per Sachs vels acozir e adobar e perfil als Sachs arecozir, Axi con seconte ellibre manoal, Assumat en una plagina en (II) cartes. .....)

Pero la participación en las tareas cotidianas de la atarazana mallorquina no se limitaba a aspectos considerados "femeninos", sino que también cooperaban en labores que requerian un mayor grado de esfuerzo físico. De esta forma, trabajando conjuntamente ambos sexos, prepararán la grada para botar o varar los buques: ... Item, pagam per moltes e diverses partides per Jornals de cauadors qui cauaren e lauraren tota la terra que era cahuta dauant lo Fondech dela Darassana e per fembres qui tiraren la terra a ops de fer lescar ales dites Galeas axi con se conten per menut $e$ per partides el libre manual e asumat en $l^{a}$ plagina en XXXXV cartes.........XXII libras, XVIIII sous. ${ }^{24}$ Item, pagam aomens cauados eafembres per esplanar lescar, eabastayns per tirar neclas alescar, e que pagam an bernat belot per loger de neclas eomens de loger quins aydaren lo leny menor atrer e per altres mecions fetes per los dits lenys axi con se conte el manuall easumat en uan plagina en VIII cartes. IIII libras, XVIIII sous, IIII ${ }^{25}$.

\section{CONCLUSIONES}

La función de la mujer en el comercio marítimo medieval continúa siendo desconocida. Sin embargo, la documentación conservada nos permite vislumbrar un rol mucho más activo del que los estudios tradicionales dejan ver, por lo que sería conveniente profundizar en su análisis. En estos breves retazos, las hemos visto actuar en distintos aspectos relacionados con la actividad económica, lejos del encasillamiento que la historiografía parece empeñada en asignarles, y bien implicadas en el desarrollo del comercio mallorquín.

23 A.R.M. R.P-1103, fol. 9r: ...Assi comensen les (messions) fetes per derassana en veles adobar, arbres eentenes e ancores e yors, exarcies decanem e derba e rems fer a adobar, armes comprar e adobar; Botes e barils obs de Galees comprar. e adobar Galees deportopi, ensen remps, almol amenar e delmol, sens rems, tornar aportopi; e Galees almol hormejar, homens logar e totes altres mecions fer quis pertanyen asaluament de Galees e deles exarcies..

24 A.R.M. R.P.3051, fol. 52v.

25 A.R.M. R.P-3051, fol. 58r. 


\section{BIBLIOGRAFIA}

BatLle, Carmen. Noticias sobre la mujer catalana en el mundo de los negocios (siglo XII). En "El trabajo de las mujeres en la Edad Media Hispana", p. 201-221. Asociación cultural AL-MUDAYNA. Madrid, 1988.

Hinojosa Montalvo, José. La mujer en las ordenanzas municipales en el Reino de Valencia durante la Edad Media. En "Las mujeres en las ciudades medievales" Actas de las terceras jornadas de investigación interdisciplinaria. Seminario de estudios de la mujer. Universidad Autónoma de Madrid, 1984.

MORANT, Isabel. Historia de la mujeres e historia: innovaciones y confrontaciones. En "Historia a Debate", tomo III, pp. 293-304. Actas del II Congreso Internacional "Historia a Debate" celebrado del 14 al 18 de Julio de 1999 en Santiago de Compostela. Carlos Barros, editor. 2000.

TUDELA VILLALONGA, Lluis. Les dones dels mercaders genovesos i mallorquins a la primera meitat del segle XIV. MAYURQA, n. 28, p. 225-239. Palma de Mallorca, 2002.

VIERA, David y PIQUE, Jordi. La dona en Francesc Eiximenis. Biblioteca de Cultura Catalana, n. 66 . Ed. Curial. Barcelona, 1987. 